\title{
Chapter 3 \\ Framing the Conversation: The Role of the Exhibition in Overcoming Interdisciplinary Communication Challenges
}

\section{Susan Liggett and Mike Corcoran}

\begin{abstract}
The role of the exhibition in overcoming interdisciplinary communication challenges is explored. The meaning of interdisciplinary communication is established, alongside the challenges it brings, where their route causes lie and the extent to which the exhibition is equipped to address them. It concludes that exhibitions of a particular nature are uniquely equipped to facilitate better quality interdisciplinary communication in certain contexts, but that further work is needed to develop mature curatorial models to facilitate this. A structure for such models is proposed, alongside the steps that now need to be followed if exhibitions are to fulfil this untapped potential at the interface of art and technology.
\end{abstract}

Keywords Curatorial model $\cdot$ Communication $\cdot$ Exhibition $\cdot$ Interdisciplinary $\cdot$ Art $\cdot$ Technology

\subsection{Introduction}

Interdisciplinary solutions are becoming the lingua franca in the discussion of the global challenges we face in the twenty-first century. Whether it's the education of our young people, the care of our elderly, the fight against disease or the protection of our environment, the answers will not be found in any one place. Rather, progress relies on the coming together of diverse expertise and perspectives, and the shared commitment of a vast number of individuals, unified by a common goal.

At the meeting point of art and technology, this is felt more sharply than perhaps anywhere else. The 'fourth industrial revolution' has blurred the boundaries between man and machine, with the designer's role fundamental in managing the interface between the two. Furthermore, artists have ever-increasing responsibility for bringing the challenges of our times to the masses in ways which are tactile, accessible

S. Liggett $(\bowtie) \cdot M$. Corcoran

Wrexham, Wales

e-mail: s.liggett@glyndwr.ac.uk

M. Corcoran

e-mail: mike@macorcoran.com

(C) The Author(s) 2020

R. Earnshaw et al. (eds.), Technology, Design and the Arts-Opportunities and Challenges, Springer Series on Cultural Computing, https://doi.org/10.1007/978-3-030-42097-0_3 
and engaging to all: driving political and social agendas. Conversely, technology is opening up endless new ways to create, display and interact with art, spawning new branches of artistic practice, and changing our perceptions of what art is and what it can achieve.

Communication is central to the success of any group, working towards any objective. When groups are interdisciplinary, subject matter is technical, stakeholders are global and success is critical, this presents unique challenges. However, in spite of this, the nuance of communication in such contexts is still poorly understood. Exhibitions are a unique communication tool. Their potential as a means to support better quality communication amongst interdisciplinary teams is apparent; yet this potential is under-investigated. The processes by which it could best be realized are ill-defined, and the models and tools required to facilitate the execution of such processes are absent.

Here, a forensic examination of communication amongst interdisciplinary arttechnology teams is undertaken to establish the root causes behind the challenges which arise and the extent to which exhibitions are equipped to provide solutions to them. Assumptions are tested against the experiences of the principal actors in such communications and the way forward is posited, with proposals set out for the further work required if exhibitions are to fulfil their potential in improving interdisciplinary communication: for more people, more of the time.

\subsection{Defining Interdisciplinary Communication}

\subsubsection{Defining Interdisciplinary}

Before we identify interdisciplinary communication challenges, we must first establish what is meant by interdisciplinary and communication.

The terms interdisciplinary, multidisciplinary and transdisciplinary and applied inconsistently, and used interchangeably by many [1]. However, the National Academies provide the following working definition of interdisciplinary research: "a mode of research by teams or individuals that integrates information, data, techniques, tools, perspectives, concepts, and/or theories from two or more disciplines or bodies of specialized knowledge to advance fundamental understanding or to solve problems whose solutions are beyond the scope of a single discipline or area of research practice" [2].

It is in this sense that interdisciplinary will be referred to herein: referring to the coming together of teams or individuals from two or more bodies of specialized knowledge in research, or otherwise. 


\subsubsection{Defining Communication}

In order to precisely identify communication challenges, it is helpful to adopt a simple conceptual model of human communication which clearly delineates each of its component parts. The Shannon-Weaver Model [3] was developed by Bell Laboratories engineers Elwood Shannon and Warren Weaver in the 1940's. It is widely regarded as the forerunner of modern communication studies and provides such components. Shannon and Weaver extrapolated a general theory of communication from the process by which radio technologies function and identified five components of all communications, as represented in Fig. 3.1.

An Information Source produces the message which is to be communicated. A Transmitter encodes that message into appropriate signals for transmission. A Channel provides the medium through which signals are transmitted (and as signals propagate through this Channel, they are vulnerable to 'Noise' which can block, interrupt or distort signals). A Receiver decodes the signal to reconstruct the original message, and a Destination is an individual, group or entity for whom the message is intended.

Consider saying hello to a friend as you walk down the street. You, or perhaps we should say, the appropriate regions of your brain, are the Information Source, taking the decision to transmit the message, 'Hello!'. Your lungs, vocal folds, tongue and mouth are the Transmitter, working together to produce the appropriate sound waves and air is the Channel which carries these sound waves between you and your friend. Passing buses, sudden gusts of wind and much else can provide noise to distort the message at this stage, before your friend's ear receives the sound waves transmitted, and their brain converts and translates them to reveal their intended meaning. The message has now reached its intended destination and your friend

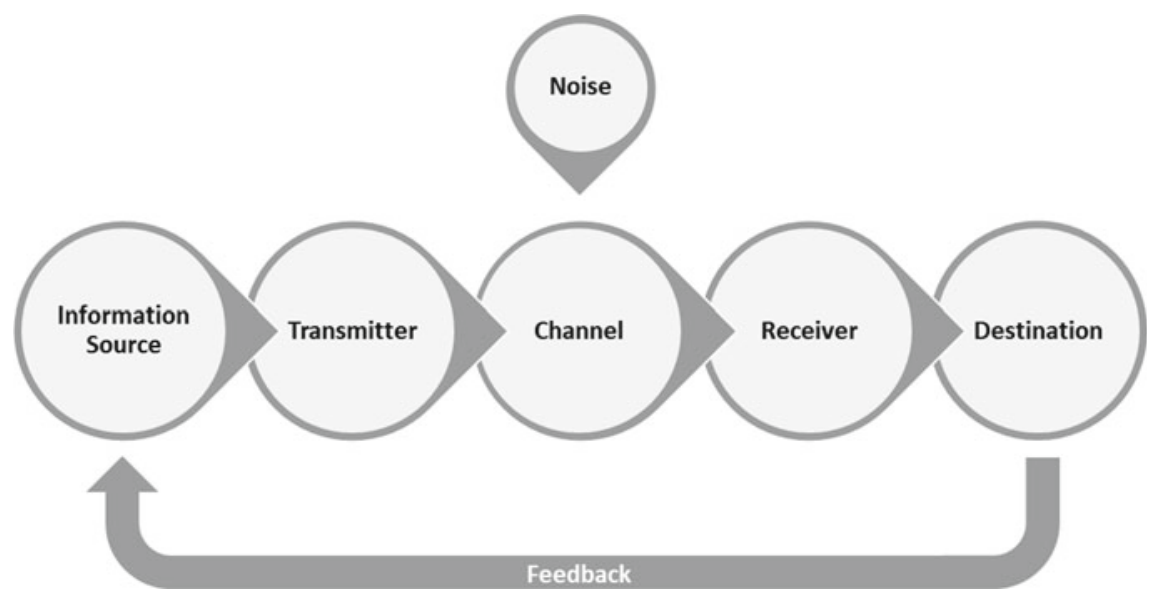

Fig. 3.1 Shannon and weaver's five component parts from which all communications are comprised. Copyright (C) M. Corcoran 2019 
responds appropriately, perhaps with a wave in your direction. Through this new transmission (and feedback), the iterative process begins again.

\subsubsection{Defining the Principal Actors in Interdisciplinary Communication}

Alongside a conceptual model of communication, it is also crucial that we have an understanding of who the principal actors (in Shannon and Weaver's language, the Informational Sources and Destinations) in interdisciplinary communications typically are. We propose the following groups, as shown in Table 3.1.

With working definitions of interdisciplinary and communication established, alongside an understanding of who such interdisciplinary communication occurs between, we can now consider the challenges of interdisciplinary communication and their direct relationship to activities involving artists and technologists.

\subsection{Interdisciplinary Communication Challenges}

Shannon and Weaver proposed that, considering the five parts from which all communication is comprised, three fundamental types of communication challenge can arise:

Table 3.1 The principal actors interdisciplinary communication

\begin{tabular}{l|l}
\hline Principal actor & Definition \\
\hline Specialists & $\begin{array}{l}\text { The individuals and teams who carry out the work. They possess knowledge of } \\
\text { one or more technical discipline and are unified by a common set of objectives }\end{array}$ \\
\hline Benefactors & $\begin{array}{l}\text { Those who sponsor the work. This includes philanthropic funders, public } \\
\text { bodies and private investors }\end{array}$ \\
\hline Beneficiaries & $\begin{array}{l}\text { Those who reap the rewards of the work, including governments, private } \\
\text { companies and proportions of the general public }\end{array}$ \\
\hline Publics & $\begin{array}{l}\text { A meta-group that include all those within a given population who are } \\
\text { stakeholders in an interdisciplinary activity, either as specialists (contributing } \\
\text { their insights with respect to a particular problem), Benefactors (through the } \\
\text { public funding of interdisciplinary work through taxation or others) and } \\
\text { Beneficiaries (in virtue of an activity's impacts). Often Publics will hold } \\
\text { multiple roles simultaneously }\end{array}$ \\
\hline Translators & $\begin{array}{l}\text { Facilitating communication between two or more of the aforementioned } \\
\text { groups in any combination. For example, they may assist specialists in } \\
\text { engaging with Publics, Benefactors in evaluating Beneficiaries, or Publics in } \\
\text { lobbying Benefactors }\end{array}$ \\
\hline
\end{tabular}


1. Technical Challenges: relating to how accurately messages can be issued.

2. Semantic Challenges: relating to how precisely meanings can be conveyed.

3. Effectiveness Challenges: relating to how successful received messages are in bringing about their desired effects.

\subsubsection{Communicating Accurately}

Technical challenges arise due to the many different means artists and technologists have to adopt to get their messages across.

The typical interdisciplinary communication involves multiple information sources, and multiple Destinations. Diverse information is shared in all directions: both within specialist interdisciplinary teams, and between those teams and their Benefactors, Beneficiaries, Publics and Translators. Every group (and every actor within every group) has their own preference with respect to transmitting and receiving information (some like to write or talk, others like to draw or experience and so on), and information tends to go through many iterations of transmission and reception on its journey between actors, highly subject to noise and interference.

This is especially true when artists and technologists work together. Consider what it takes for the latest research in machine learning to reach the desk of the fine art professor or for the biomedical scientist to encounter theories in the teaching of painting. Consequently, before the insights of one actor enter the consciousness of another, they may be transmitted through many cycles of performance, discussion, publication or otherwise, with each increasing the probability of degradation, mistranslation and dilution of the overall message [4]. Even before interdisciplinary teams have been constructed, the basis for their very construction can run the risk of being underpinned by inaccurate information.

\subsubsection{Communicating Precisely}

Semantic challenges arise due to the difficulty of explaining things in a simple way which artists, technologists and everyone they engage with can understand.

There is no universally understood language amongst the principle actors in interdisciplinary activities. As Derrick et al. observe, "Scientific disciplines have different cultures, languages, and standards" [5] and the same can be said of non-scientific disciplines. The vocabulary and reference points of specialists are highly technical, and a deep understanding of issues is often contingent upon a contextual understanding of the wider subject, not always available to every specialist within an interdisciplinary team. This is particularly true when artists and technologists come together. Their respective disciplines having long been siloed into what CP Snow famously characterized as 'The Two Cultures' [6]. 
This problem is only compounded as specialists (of any discipline) look to communicate to their Benefactors, Beneficiaries and Publics. Translators can assist, but ultimately, communication is often heavily reliant on metaphor and simplification, inevitably impacting on the precision with which information can be shared, and preventing much information from being shared at all.

\subsubsection{Communicating Impactfully}

Effectiveness challenges arise due to the many different things artists and technologists have to simultaneously achieve when they communicate.

Often, a single communication will have multiple Destinations and multiple diverse objectives. As communication takes place amongst specialists within an interdisciplinary team, the outcomes and impacts of such work are simultaneously subject to the scrutiny of Benefactors, Beneficiaries and Publics, each with diverse needs and expectations. To achieve such objectives can be 'complex, nuanced and highly resource intensive' [4]. When it is simply not possible to tailor the perfect transmission for each target Destination, a 'one size fits all' approach can be considered as an alternative, but in striving to please all, there is an inherent risk of not pleasing any.

Taking into consideration the main components of communication, the fundamental types of communication challenge posited by Shannon and Weaver, and reflecting on each in the context of the principal actors in interdisciplinary communication, especially artists and technologists, we can conclude that such communication is a challenge because:

1. Technical challenges lead to the accuracy of information shared being impeded by the many different ways and means required to communicate.

2. Semantic challenges lead to the precision of information shared being impeded by the lack of common vocabulary and reference points amongst the principle actors who are communicating.

3. Effectiveness challenges led to the impact of communication being impeded by the multiple objectives it is required to serve.

In transitioning from an understanding of the challenges which lie at the heart of interdisciplinary communication to an understanding of the ways in which these challenges can be overcome and the role of the exhibition in facilitating this, it is essential to gain a deeper appreciation of the validity of our conclusions above. This includes the extent of their generalisability, and the contexts in which they most strongly apply. This starts through dialogue with those with lived experience of interdisciplinary collaboration from a variety of different perspectives. 


\subsection{Interviews with Interdisciplinary Practitioners}

The following perspectives are derived from interviews conducted with various experts and specialists in the domain of this research. That is, those who are primarily responsible for the management and administration of interdisciplinary projects and activities, and the communication that comes with it. Their thoughts and experiences are summarized below.

\subsubsection{The Specialist Technologist's Perspective}

The specialist technologist's perspective was offered by a software engineer working predominantly in the rendering of digital holograms.

This involves communicating primarily with:

- Artists (Benefactor/Beneficiaries): including both independent artists and commercial customers looking for holographic solutions within their work and

- Optical engineers (Specialists): in order to understand and provide feedback on the strengths and restrictions of working in the medium.

Communication with fellow technical specialists (i.e., software and optical engineers) tends to be the most straightforward. This is in part thanks to the common vocabulary and reference points these specialists share, and the readily quantifiable nature of the information they typically share (for example, the technical parameters of the hologram printing equipment). For communication of this nature, communication tools such as email are very effective, allowing for precise information to be shared back and forth asynchronously.

Conversely, communication between artists and engineers is more challenging, because there is no common vocabulary and the information being shared is typically subjective and qualitative. Engineers and artists are often exploring the 'art of the possible', and the broad objective which the creative work is attempting to realize. In these circumstances, live and iterative communication, supported by visual aids, can prove effective.

With regards to exhibitions, they can play a valuable role in setting the context for discussions between any actors, alongside demonstrating the nature and potential of the technology, especially when bringing such state-of-the-art technology to general public audiences.

\subsubsection{The Specialist Artist's Perspective}

Jessica Lloyd Jones, an artist who exhibits internationally combining elements of art, science and technology provided the specialist artist's perspective. Lloyd Jones' work 


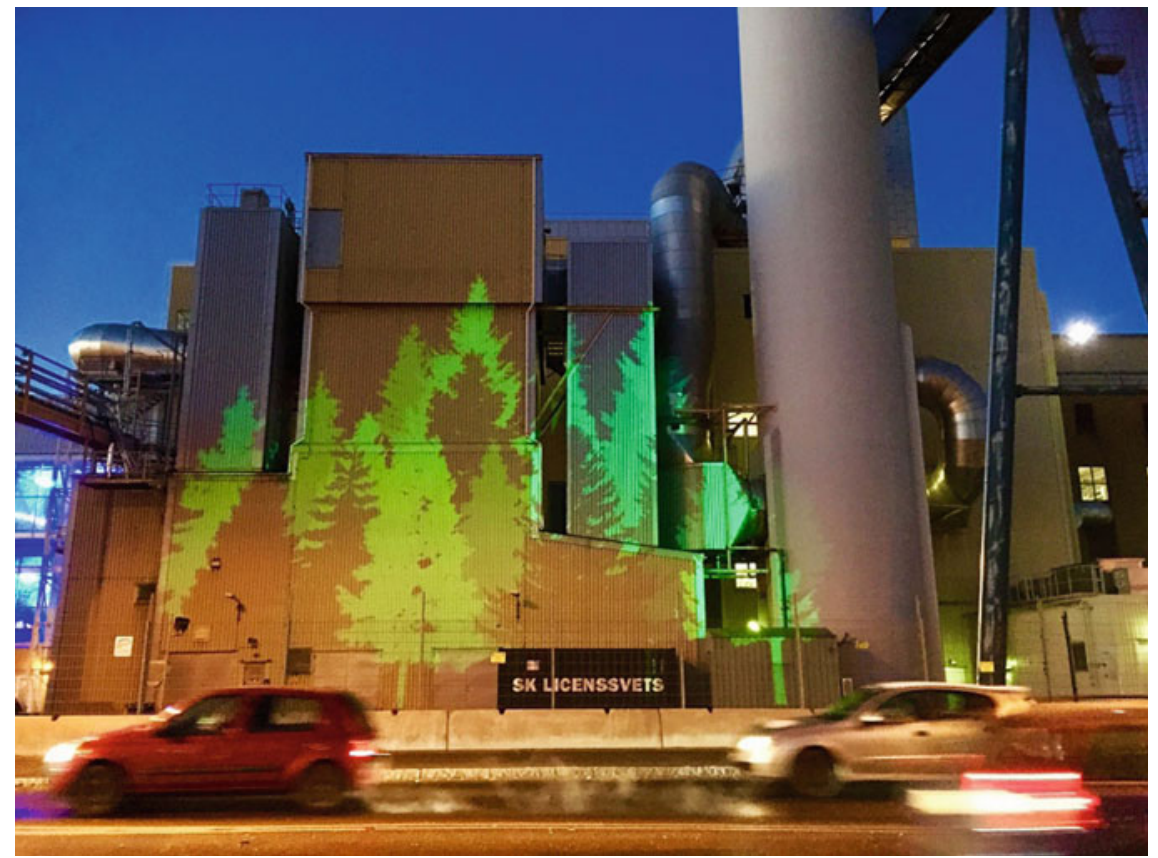

Fig. 3.2 Jessica Lloyd Jones 'Forest Fuel' 2018, permanent projection onto a biomass plant, Gothenburg, Sweden. (Copyright () J. Lloyd Jones 2018) included by permission

explores concepts of energy, matter and natural phenomena through the interaction of materials and light through installations, projection and sculpture created from a variety of materials and processes. An example of this work is shown in Fig. 3.2, where a digital image is projected on to a biomass plant in Sweden.

Producing works of this nature requires effective liaison with a wide group of people. These include project managers, funders and commissioners (Benefactors), technologists, engineers, architects, fabricators and design teams (Specialists) and curators (Translators).

Internal communication between these people is, in part, influenced by the time sequence in which the various actors signed-up to the project. For example, commissioners and project managers will often be in place before the artist becomes involved, whereas others (for example, fabricators) can only be selected by the artist once a project has commenced. The structure, roles and responsibilities of a team will often be assigned in line with the common goals identified between the artists, technologists/scientists and the other stakeholders involved, with ultimate management responsibility depending on who instigated the project. Maquettes and visualizations provide useful aids here, illustrating ideas and concepts to stakeholders and potential stakeholders.

External communication to an artwork's intended audiences must simultaneously speak to many diverse individuals. Those with an active interest in the arts are the 
easiest to reach as they tend to be more open-minded and curious. This is especially true when the information to be communicated is broad-based and thematic, for example, communicating ideas about energy, beauty, innovation or creativity. When audiences have little knowledge, understanding or exposure of the arts, this can directly impact upon enthusiasm, open-mindedness and curiosity, and subsequently on the success of the communication which follows.

No one tool is the secret to successful communication, with the most effective means of communication depending on the context, though when engaging in a crowded market-place, novelty can be key. Audiences are more likely to be engaged if the artwork presents something they haven't seen before.

\subsubsection{The Benefactor's Perspective}

The benefactor's perspective was offered by a senior manager in a UK-based public services innovation foundation, often delivering projects bringing together artists, technologists and public bodies.

Audiences in communication in this environment are diverse. This is not only a diversity of organisations, but a diversity of individuals within each organisation, which communication must be sensitive to. However, across all audiences, the communication of 'facts and figures' tends to be easier than the communication of 'concepts and ideas,' thanks to:

- The clear and unambiguous nature of the information: usually concerning who, what, when, where and how;

- The clearly delineated audiences for the communication: with eligibility criteria defining the types of organisation for whom the information is relevant;

- The simple and well-established channels through which communication is shared: for example, application forms to access funding and;

- The simple feedback loops associated with these channels: for example, via workshops, emails and phone calls.

Conversely, communicating concepts and ideas, especially the learning arising from projects, is generally more challenging. Points can be nuanced and subtle; the learning potentially applicable to any audience and the channels through which such learning is shared are less well-established resulting in feedback loops which can be vague or non-existent.

Additional challenges can arise due to the differing organisational cultures in place between partners in a given project (especially those bringing academic, public and private sector partners together). Each may have different reasons for being involved in the project, and these need to be taken into account. At the same time, this diversity can be a strength of the project because it can lead to innovation and new ways of viewing the project and its outcomes, which can add value to the original concept.

Exhibition is a tool used regularly to communicate concepts and ideas, present the outcomes achieved and learning gained through collaborative projects and to foster 
multiple conversations simultaneously between many partners. It is often easier to show someone something than tell them about it. The innovation foundation often use 'pitching exhibitions' to invited audiences of key stakeholders at targeted events, which they find to be a highly effective method.

\subsubsection{The Translator's Perspective}

The translator's perspective was offered by a public engagement manager at a leading biomedical research facility. A permanent gallery at the facility, along with an accompanying public engagement outreach programme, aims to generate excitement and interest in discovery of science, human health and wellbeing: through artistic means and otherwise.

Exhibitions have to cater to many audiences, marrying rich content for high science capital peers (including those researchers based at the facility) simultaneously with that for a low science capital lay-public. Often, they also need to cater to visitors from different countries (with different cultural backgrounds), politicians, distinguished guests, as well as the accompanying press: simultaneously and seamlessly.

To bring these exhibitions together requires close communication with and between:

- Public engagement and education teams (Translators)

- Press and marketing teams (Translators)

- The organisation's scientists (Specialists): including those directly involved in each exhibition, and the wider community of those working in the organisation.

- Exhibition funders (Benefactors).

- Contractors (Specialists) of diverse backgrounds and specialisms.

- Focus groups (Publics and Beneficiaries) contributing to each exhibition's relevance and accessibility.

- Peers (Specialists and Translators): working in museums, galleries, and other science-communication environments, sharing best practice, knowledge and experience.

In practice, the planning of each exhibition can require communication with a diverse group of 30-40 people.

Internal communications are managed with a high degree of structure. An Internal Advisory Panel of senior managers and key stakeholders communicate the exhibition's broad themes and messages. It also translates these into the wider strategic direction and brand identity that the organisation is seeking to achieve. This helps to build trust-so that the senior managers and key stakeholders feel confident in the processes being followed and the expected outcomes. An exhibition steering committee comprised of colleagues with a variety of roles across the organisation can provide feedback on various aspects of the exhibition plans. A project group is normally required to discuss the practicalities of bringing each exhibition together and 
meeting the needs of all stakeholders within the constraints of the space available. It is also important to maintain communication with any external teams of contractors and keep track of timelines, deliverables and issues as they arise. Communication within these structures is aided by video conferencing, formal presentations, document sharing and Gannt charts, but the most effective method is often to communicate face-to-face (or via video conference for more remote collaborators).

Communication is most effective when it is between individuals who all work in the same context, with the same strategy, and the same audience. The greatest challenge is when the time is limited, audiences are disparate, and a contextual understanding is absent, communication is far more complex.

With regard to the content of the exhibitions, to achieve their intended impact of making a tangible impact to the feelings and perceptions of their audiences, consideration needs to be given to the diverse ways in which they may be interpreted by different kinds of audiences, particularly where advanced or specialist subjects are involved. There is also a distinction between the explicit information the exhibition communicates directly and the implicit communication of its values, character and context. The latter can be assisted, amongst other things, by the atmosphere created by the exhibition and the personnel who bring it to life. Both are needed if the exhibition is to be successful.

\subsubsection{The Combined Perspective}

In many cases, one individual may take on multiple communication roles in their work.

Such a combined perspective was offered by Jamie Harris, founder of Elder3D, a company specialising in making artworks affordable to the masses through utilization of cutting-edge 3D design and manufacturing technologies (Fig. 3.3). Harris is a

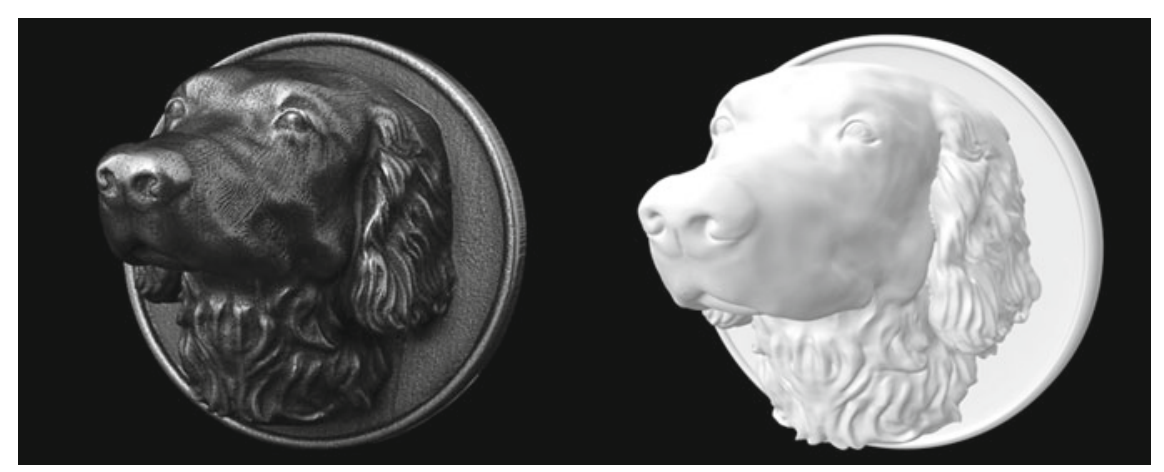

Fig. 3.3 A metallic 3D Print (Left) and its supporting digital 3D Model (right). (Copyright ( $)$ J. Harris 2019) included by permission 
Specialist in both artistic and technological practices, a Benefactor commissioning work from his international network of associate artists and technologists and a Translator, ensuring the wants and needs of his customers are always catered to.

In this context, understanding and trust are the basis for successful communication. Artists and 3D print specialists have to understand that the quality of the final product is dependent on the quality of information provided initially. The print specialists have to understand every detail in the brief and customers have to understand what is and what is not possible within the limitations of the technology.

Communication is easiest when the information shared is quantitative and unambiguous (for example, specifying the material and dimensions for a 3D print run) and when it is between actors who speak a common language, sharing common skills and experiences. Conversely, when the information to be shared is nuanced and qualitative (for example, when a family wishes to convey the 'spirit' of a person in a portrait) and when commonality of language is missing, communication is a challenge. Commonality lies in the shared experience of actors, but also on the number of individuals comprising each 'Information Source'. When a customer artist or printer is in practice, and when decisions are made by committee, information can quickly become confused, unclear or contradictory.

Trust is a prerequisite to understanding allowing honest and open communication to take place customers must trust that their vision can translate into a high-quality product, and artists must trust that a printer can bring their designs to life, print specialists must trust that the designs will be suitable for 3D printing in the specified materials and so on. Therefore, establishing a strong rapport and a personal relationship with everyone is essential to the business.

With regards to exhibition, showing the actual work is often the best way to communicate because people can touch and handle the work. This creates excitement, promotes questions and produces a better understanding.

\subsubsection{An Interdisciplinary Communication Tool Wish List}

In Sect. 3.3 we proposed that the challenge of interdisciplinary communication was rooted in the many different ways and means required to communicate (impairing accuracy), the lack of common vocabulary and reference points amongst the principal actors communication is between (impairing precision), and the multiple, simultaneous objectives such communication is often required to serve (impairing impact).

The views of those with direct experience of working in these environments certainly do not contradict these conclusions. Rather, we observe many instances of these challenges in action. We also gain a deeper insight into how these challenges arise, the other challenges they are closely related to and how such challenges might be addressed. 
Table 3.2 An interdisciplinary communication tool 'wish list'

\begin{tabular}{|c|c|}
\hline \multicolumn{2}{|r|}{ An interdisciplinary communication 'wish list' } \\
\hline \multicolumn{2}{|r|}{ More accurate communication (to overcome technical challenges) } \\
\hline 1 & Reduce the total number of channels needed to communicate messages \\
\hline 2 & Reduce the quantity and improve the quality of Transmitters/Receivers \\
\hline 3 & Reduce the risk of noise within multiple communication-feedback cycles \\
\hline \multicolumn{2}{|r|}{ More precise communication (to overcome semantic challenges) } \\
\hline 4 & Bring clarity to open ended, subjective and qualitative messages \\
\hline 5 & Bring shared language, reference points and context to messages between diverse actors \\
\hline 6 & Bring clarity to single messages compiled by multiple actors \\
\hline \multicolumn{2}{|r|}{ More impactful communication (to overcome effectiveness challenges) } \\
\hline 7 & Provide simultaneous communication of multiple (implicit and explicit) messages \\
\hline 8 & Build trust and rapport amongst the principle actors in communication \\
\hline
\end{tabular}

Taking all of this into consideration, we propose a simple 'wish list' (see Table 3.2) of outcomes for any tool which could make interdisciplinary communication involving artists and technologists easier to facilitate.

With a wish list for an interdisciplinary communication tool established, we can assess the extent to which the exhibition has the potential to be such a tool. This can be done by considering what exhibitions are and how well aligned (and potentially impactful) they are with respect to each of our desired outcomes.

\subsection{The Exhibition as an Interdisciplinary Communication Tool}

\subsubsection{Defining Exhibition}

Exhibition is a broad and all-encompassing term. It may refer to any "event at which objects are shown to the public, a situation in which someone shows a particular skill or quality to the public, or the act of showing these things" [7], and it is this general sense that exhibitions are discussed here. This definition excludes private and informal day-to-day interactions. Furthermore, when we discuss exhibitions herein, we refer primarily refer to them in their most common temporary and ephemeral form, as opposed to permanent installations and collections.

Our wish list (Table 2.2) is composed in the language of the Shannon-Weaver Model and so before we can assess exhibitions against each of its desired outcomes, we must first ensure we can describe exhibitions in a consistent way. For virtually any exhibition, in any form:

- The ideas of the exhibiting artist and/or the curator serve as the Information Source. 
- The artworks, in their particular arrangement, are the Transmitters of these ideas.

- The exhibition environment is the Channel through which these ideas pass.

- The senses of the exhibition's audiences are the Receivers of these ideas, and

- A given subset of the exhibition's total audience is the intended Destination for these ideas.

Furthermore, as done so throughout Sect. 3.4, the principal stakeholders in exhibitions can be simply mapped to the principal actors in interdisciplinary communication which we have defined as follows:

- Specialists: the exhibitors and/or the exhibition's curator.

- Benefactors: the sponsors, institutions or others who fund the exhibition.

- Beneficiaries: the audiences who are the intended 'Destination' for the exhibition's messages.

- Publics: formed from some combination of the above.

- Translators: curators and exhibition assistants.

With these definitions in place, our desired outcomes can now be assessed in turn.

\subsubsection{Assessing Impact}

Exhibitions can address technical challenges, leading to improved accuracy in communication, by reducing the number of Transmitters, Receivers and Channels, needed to communicate information. Each Information Source has one Transmitter, an exhibit, fewer transmitters implies fewer receivers to get a message to its intended destination, and the exhibition provides the sole Channel for communication. Furthermore, its structured nature allows for multiple stable feedback cycles, providing a constant background, providing a general context against which all further communications can be located.

Exhibitions can address semantic challenges, leading to improved precision in communication, by front-loading the communication process with the information which does not require the use of technical and discipline-specific vocabulary and reference points to understand: that is, the general qualitative messages that lie behind the technical detail. The exhibits themselves become the common, visual and tactile transmitters used to communicate these messages, to actors who do not require any technical knowledge to receive them. By precisely communicating the general messages behind work at the earliest opportunity, subsequent discussion of that work (through symposia, informal meetings, exhibition guides or otherwise) and all that follows thereafter, becomes much easier, whether between one induvial or many.

Exhibitions can address effectiveness challenges, leading to improved impact in communication, by simultaneously communicating to multiple and diverse actors. For some (for example, Publics), a general sense of 'what the work is about' is the desired outcome. For others (for example Benefactors), this general sense is a precursor to a desired outcome of a more detailed understanding why this work is 
needed, at this time. For Specialists, this detailed understanding is also a precursor to understanding who they should work with, how they should work with them, and where they need to widen their technical understanding (or recruit the support of translators) to facilitate this. Each of these can use the same core set of transmitters, the exhibits, and the sole channel of the exhibition. The exhibition can be curated so as to allow multiple and mutually compatible routes through it. Some may view the exhibition online; others may view it in person. Some may use an exhibition guide; others take a guided tour. Some may participate in symposia; others use it as a venue for meetings. Some may spend only minutes in the space; others hours or days. All of this can happen at the same time. There is no direct analogy for this flexibility with a research paper, a seminar or many of the other traditional tools of interdisciplinary working.

Not only that, but by putting an initial emphasis on common thoughts and feelings, in an environment which lends itself to being inclusive, social and highly creative, the exhibition plays a role in building the relationships, trust and rapport that are prerequisite to good communication, in a space which can entice and motivate new audiences to join in the fun.

\subsection{A New Curatorial Model}

The potential of the exhibition to provide solutions to interdisciplinary communication challenges, as outlined in Sect. 3.5, is clearly encouraging, but our discussion so far is insufficient in and of itself. To support meaningful action and generate real outcomes, this potential must be pinned down and converted into the clear guidance required by exhibition curators and those responsible for making interdisciplinary communications work. Our observations need consolidating into a model, stipulating what sort of exhibitions, will deliver what sort of outcomes, for what sort of audiences, and how.

To date, there is no off-the-shelf conceptual model describing the functions of the exhibition as an interdisciplinary communication tool, and perhaps this should not be surprising. Exhibition and curatorial studies, and the discourse and theoretical models which underpin them, do not share a common genus with communication theory. They have evolved divergently, fuelled by different motivations, setting out to solve different problems and thinking about those problems in different ways.

The Show-Talk-Do Model [4] (Fig. 3.4) describes the process by which high-level analysis of opportunities for research collaboration, alongside simultaneous nonexpert engagement, can be achieved in environments which are rich with technical information and highly interdisciplinary. It contains three simple steps, which can be summarized as follows:

1. Show It: Focuses on initial organic reactions. A public exhibition presents the organic products of the ongoing research of participating researchers, from across a variety of disciplines and interdisciplinary areas. Exhibits may include (but 


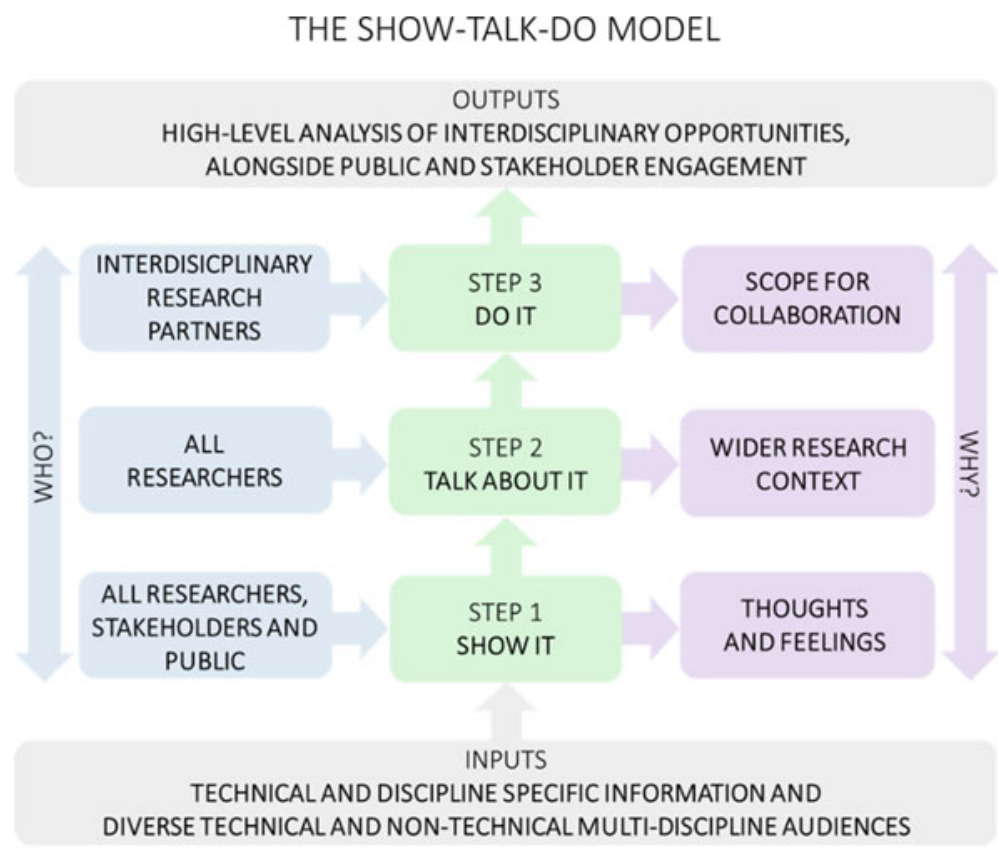

Fig. 3.4 The Show-Talk-Do Model for multi-audience engagement (@ M. Corcoran and Liggett. S. 2018)

are not limited to) artworks, performances, documentation of practical research activities, visualization of 'live' research data and interactive demonstration of a process or procedure under development.

2. Talk About It: Focuses on developing contextual understanding. A symposium (or similar) takes place, where exhibiting researchers discuss with one another the context in which their exhibit (and by proxy, their research) is situated. This discussion should be structured (in accordance with pre-defined rules) and be located within the exhibition itself.

3. Do It: Focus on technical understanding and action. Research papers (or similar) are made available to researchers, and where synergy has been identified through Steps 1 and 2, the scope for research collaboration can be discussed.

The exhibition itself lives within Step 1. Open to all (with Specialists alongside Benefactors, Beneficiaries and Publics in their various capacities), its primary role is to build common understanding on the level on thoughts and feelings, free from any pre-convinced ideas and prejudices:

The exhibition should be curated as to allow each work to be considered in isolation, as well as all considered in combination ... Exhibit labels (if used) should not refer to exhibitors' subject specific disciplines, their academic status or seniority, and should provide no information about the research presented. The exhibition should be made open and accessible to all the defined project's stakeholders, including researchers, funders, policy makers, and the general 
public. Visitors (including the exhibiting researchers themselves) should be encouraged to explore the exhibition with an open mind and reflect upon their spontaneous thoughts and feelings regarding the individual works and the combination [4].

When principal actors in communication have connected on an emotional level, they are then best disposed to discuss the wider context in which research is being undertaken (Step 2), and only after that, they are best disposed to engage in a labour intensive and technical discussion regarding the scope of future collaboration (Step 3 ). These things can take place within the same exhibition environment, and over the same time period, but it is only when they are in this sequence, that the process will achieve its desired results. Also, different principal actors will be required to progress through different numbers of steps. For Beneficiaries, Step 1 may be enough, being inspired, challenged, provoked or otherwise by the works presented to them. For many Specialists, Step 2 will be enough, understanding their peers' motivations and objectives, and only for those Specialists committed to working closely together (in virtue of what they have learned through Steps 1 and 2), are the challenges of getting to know the technical details of one another's practice (Step 3) required.

Show-Talk-Do was developed with a focus on interdisciplinary research. However, this structure is applicable to communication in interdisciplinary contexts more broadly. It provides a simple conceptual model concerned with communication, considering the exhibition as a communication tool alongside all others, and provides a helpful foundation for assessing the potential impact of the exhibition to bring about particular outcomes in particular circumstances, well aligned with the interdisciplinary communication challenges that have been identified.

\subsection{Conclusions}

This work has sought to establish what role, if any, the exhibition had in overcoming interdisciplinary communication challenges.

The adoption of a conceptual model of communication identified that the challenges that arise can be grouped in terms of accuracy, precision and impact. Through discussion with those with lived experience of these challenges, eight outcomes were identified which the ideal interdisciplinary communication tool would be able to tackle. The general ways in which the exhibition is well placed to meet these outcomes were identified. The adoption of the Show-Talk-Do Model enabled discussion on where these impacts might be most greatly felt, and how.

Therefore, the exhibition, by virtue of its open, creative and engaging nature, its simple means of communicating messages and its situation at the nexus of many audiences, does have an important role to play in overcoming interdisciplinary communication challenges. It is likely to be especially significant when:

- The actors engaged in communication are large in number and diverse in nature.

- The messages being communicated are broad, qualitative and contingent on an 'emotional/experiential' understanding. 
- The messages to be communicated are numerous and communicated to many actors simultaneously, each with their own objectives.

- Open and transparent communication amongst and between all actors is desirable.

- The communication process itself is intended to attract and grow its own audience.

The exhibition does not replace other tools. On the contrary, It is most likely to have a major impact when delivered in conjunction with other tools, not only achieving its own ends, but serving as a means to foster higher quality discussions, and ongoing research and partnerships.

However, if the exhibition is to consistently achieve these impacts and optimize its role as an interdisciplinary communication tool, there is still much work to do. We suggest the following three next steps are now required.

\section{(i) Better conceptual models}

The more mature the conceptual models, and the more articulate they can become at describing how exhibitions bring about their effects, the more useful they will become to the curators and other stakeholders in those exhibitions. Show-Talk-Do is a helpful start point, not an end. A sign of progress will be when it is rigorously scrutinized, refined and ultimately replaced, with better, more powerful models.

\section{(ii) Greater exhibition infrastructure}

It must become easier for those who want to use exhibition as a communication tool, to do so. Often, such tools are used not because they are the best option, but because they are the available option. Art and educational institutions, curators and research funders all have a responsibility to make exhibition an option: offering the expertise, guidance, facilities, resources and finance that are required to do exhibition well, and reap the just rewards.

\section{(iii) Leadership}

For exhibitions to be considered an essential communication tool, occupying the same status as a research paper or seminar in professional communication, it will require a shift in mind-set amongst the interdisciplinary community, and leaders from within that community to initiate change. Early adopters will face the biggest challenge, but each exhibition delivered will make the next one easier to deliver, and the impacts should follow closely behind.

If these challenges are met, there is no reason why the exhibition should not be the mainstay of 21 st communication practice in the age of the interdisciplinarian.

Acknowledgements The authors wish to thank all those who shared their time and expertise to contribute to this chapter, most notably those we interviewed: Tal Stokes, Jessica Lloyd Jones, Rob Ashelford, Bryony Benge Abbott, and Jamie Harris. 


\section{References}

1. Derrick, E., Falk-Krzesinski, H., Roberts, M.: Facilitating Interdisciplinary Research and Education: A Practical Guide. American Association for the Advancement of Science and the Colorado Initiative in Molecular Biology of the University of Colorado (2012) [online] Available at: http://researchdevelopment.byu.edu/wp-content/uploads/2012/12/Full Report_Creating_Interdisciplinary_Science.pdf. Accessed 23 June 2019

2. National Academies.: Committee on Facilitating Interdisciplinary Research, Committee on Science, Engineering, and Public Policy. Facilitating Interdisciplinary Research. National Academy Press. p. 2 (2004)

3. Shannon, C.A.: Mathematical theory of communication. Bell Syst. Tech. J. 27 (July and October), 379-423, pp. 623 656, (1948). Available at: http://math.harvard.edu/ ctm/home/text/others/ shannon/entropy/entropy.pdf. Accessed 5 March 2018

4. Liggett, S., Corcoran, M.: Interdisciplinary research unmasked: a new curatorial model for multi-audience engagement. Stud. Vis. Arts Commun.: Int. J. 5(1) (2018) online ISSN 2393-1221

5. Derrick, E., Falk-Krzesinski, H., Roberts, M.: Facilitating interdisciplinary research and education: a practical guide. In: American Association for the Advancement of Science and the Colorado Initiative in Molecular Biology of the University of Colorado (2012). [online] Available at: http://researchdevelopment.byu.edu/wp-content/uploads/2012/12/Full_ Report_Creating_Interdisciplinary_Science.pdf. Accessed 23 June 2019

6. Snow, C.P.: The Two Cultures, p. 3. Cambridge University Press, London $(1959,2001)$

7. Cambridge English Dictionary.: Exhibition (2019). [Online] Available at: https://dictionary. cambridge.org/dictionary/english/exhibition. Accessed 05 July 2019

Open Access This chapter is licensed under the terms of the Creative Commons Attribution 4.0 International License (http://creativecommons.org/licenses/by/4.0/), which permits use, sharing, adaptation, distribution and reproduction in any medium or format, as long as you give appropriate credit to the original author(s) and the source, provide a link to the Creative Commons license and indicate if changes were made.

The images or other third party material in this chapter are included in the chapter's Creative Commons license, unless indicated otherwise in a credit line to the material. If material is not included in the chapter's Creative Commons license and your intended use is not permitted by statutory regulation or exceeds the permitted use, you will need to obtain permission directly from the copyright holder.

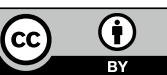

